\title{
Adaptación, aceptabilidad y pilotaje de "Usando Condón". Una intervención para incrementar la autoeficacia percibida del uso del condón masculino en adolescentes mexicanos \\ Adaptation, acceptability, and piloting of "Usando Condón". An intervention for increasing perceived male condom use self-efficacy among Mexican adolescents
}

Alma Angélica Villa-Rueda ${ }^{1}$, Erick Alberto Landeros-Olvera ${ }^{2}$, Natalia Isabel Manjarres-Posada ${ }^{3}$ y Raquel Alicia Benavides-Torres ${ }^{3}$

Palabras clave: adaptación; intervención; condón; adolescentes; uso de preservativos; educación sexual; aceptabilidad; salud

Keywords: adaptation; intervention; condom; adolescents; use of condoms; sex education; acceptability; health

Recibido en: 04-12-2019 / Aceptado en: 17-02-2020

\section{Resumen}

Introducción: Los adolescentes son un grupo vulnerable ante las posibilidades de adquirir infecciones de transmisión sexual y a experimentar un embarazo. Lo anterior puede prevenirse mediante intervenciones, basadas en teoría, sobre la autoeficacia del uso del condón masculino. Este estudio tiene como objetivo la adaptación, evaluación de la aceptabilidad y el pilotaje de una intervención para incrementar la autoeficacia percibida del uso del condón masculino en adolescentes mexicanos.

Método: Se utilizó un modelo de adaptación de intervenciones de tres etapas (evaluación, preparación e implementación) y cinco pasos, de los cuales se completó hasta al pilotaje: 1) revisión de la literatura para identificar intervenciones basadas en teoría, cuyo objetivo fuera incrementar la autoeficacia para el uso del condón; y contacto con los autores; 2) se seleccionaron dos intervenciones para el proceso de adaptación; 3) preparación del contenido de las intervenciones, para ser aplicable a adolescentes mexicanos (modificaciones de lenguaje, actividades e información); se piloteo la intervención "Usando Condón” en una muestra de 56 adolescentes pertenecientes a una preparatoria pública de Puebla, México. La aceptabilidad de la intervención se evaluó cualitativamente y se analizó mediante análisis temático. La variable resultado (autoeficacia) se evaluó mediante un instrumento específico para adolescentes mexicanos, mediante comparaciones de prueba y post-prueba.

\footnotetext{
${ }^{1}$ Universidad Autónoma de Baja California, Facultad de Enfermería. E-mail: alma.villa@uabc.edu.mx

${ }^{2}$ Benemérita Universidad Autónoma de Puebla, Facultad de Enfermería

${ }^{3}$ Universidad Autónoma de Nuevo León, Facultad de Enfermería

(C) Universidad De La Salle Bajío (México)
} 
Resultados: La intervención adaptada utilizó los componentes centrales de las intervenciones que sirvieron de base (VIH/sida; uso del condón [creencias, negociación, acceso, beneficios, consecuencia son usarlo, modelaje]; y roles de género). Se utilizaron mapas, ejemplos de historias y el modelaje del uso del condón mediante modelos anatómicos de pene. Se actualizó la información y se incluyó información sobre la anticoncepción de emergencia. Los escenarios y dosis de las intervenciones base se modificaron, así, para "Usando Condón”, se realizó una intervención de 2 sesiones de 90 minutos cada una para adolescentes mexicanos, implementada por enfermeras. La asistencia a la intervención fue del $100 \%$. La intervención fue aceptable para los participantes, quienes también sugirieron modificaciones menores a las herramientas tecnológicas de apoyo, contenido y actividades. La prueba $t$ de Student mostró cambios significativos entre las mediciones antes y después a la exposición de la intervención $(p<.05)$.

Discusión o Conclusión: "Usando Condón" puede ser implementada por profesionales de la salud previamente capacitados y en contextos similares, considerando que necesita de constante evaluación y adaptación a las características de la población. Además, los resultados preliminares sugieren que la intervención logra incrementar los niveles de autoeficacia percibida para el uso del condón masculino en adolescentes mexicanos.

\section{Abstract}

Introduction: Adolescents are a vulnerable group to acquire sexually transmitted infections and to experience a pregnancy. This can be prevented through theory-based interventions on male condom use self-efficacy. The goal of the present study is the adaptation, acceptability evaluation and pilot testing of an intervention, to increase perceived male condom use self-efficacy among Mexican adolescents.

Method: A three-stage intervention adaptation model (evaluation, preparation and implementation) compounded of five steps (completed up to the four step) was used: 1) reviewing the literature to identify theory-based interventions for increasing condom use perceived selfefficacy, and authors contact; 2) two interventions were selected for the adaptation process; 3) preparation of the interventions content to be applicable to Mexican adolescents (language, activities and information modification); the intervention "Usando Condón" was piloted in a sample of 56 adolescents belonging to a public high school in Puebla. Intervention acceptability was qualitatively assessed and analyzed through thematic analysis. The outcome variable (self- 
efficacy) was measured through a specific Mexican adolescent's scale. Self-efficacy levels were compared after the intervention (test and re-test).

Results: The adapted intervention used the core components of the interventions that served as basis for the adaptation process (HIV/AIDS, condom use [beliefs, negotiation, access, benefits, consequences of using it, modeling] and gender roles). Cognitive mapping, story examples and condom use modeling through anatomical penis models were used. Information was updated and information on emergency contraception was added. Scenarios and doses of the primary interventions were modified, resulting in "Usando Condón" a 2-sessions intervention of 90 minutes each for Mexican adolescents, implemented by nurses. The intervention assistance was $100 \%$. The intervention was qualitatively acceptable by the participants, suggesting minor modifications regrading to the technological support tools, content, and activities. Student's t test showed significant changes between measurements before and after the intervention exposition $(p<.05)$.

Discussion or Conclusion: "Usando Condón" can be implemented by previously trained health professionals and in similar contexts, considering that it needs constant evaluation and adaptation according to the population's characteristics. Additionally, preliminary results suggest that the intervention increases the male condom use perceived self-efficacy levels among Mexican adolescents.

\section{Introducción}

La adolescencia es una etapa de la vida que comprende de los 10 a los 19 años de edad (World Health Organization [WHO], 2017), marcada por los múltiples cambios que la persona enfrenta a nivel físico-biológico, social y psicológico. Por ejemplo: la maduración biológica, las transiciones en los roles sociales y las expectativas del futuro; también, la madurez psicológica que los conduce a diversas experiencias en búsqueda de autonomía, identidad sexual y social, y con ello a mayor vulnerabilidad y exposición a situaciones de riesgo para la salud (Sawyer, Azzopardi, Wickremarathne \& Patton, 2018). De los resultados adversos más pronunciados en este grupo de personas, se encuentran la adquisición de infecciones de transmisión sexual (ITS), como el Virus de Inmunodeficiencia Humana (VIH) y el embarazo, relacionados a conductas sexuales de riesgo como el uso inconsistente del condón (WHO, 2017). 

percibida del uso del condón masculino en adolescentes mexicanos

Las personas de 10 a 19 años de edad componen el grupo etario más grande en la sociedad mexicana (Instituto Nacional de Estadística y Geografía [INEGI], 2017). En México, la edad mediana del debut sexual en mujeres adolescentes es de 17.5 años y la tasa de fertilidad para mujeres adolescentes entre 15 a 19 años es de 70.6 nacimientos por cada 1000 mujeres (Gobierno de la República, 2016; INEGI, 2018a; Organización para la Cooperación y el Desarrollo Económico [OECD], 2019). En Estados Unidos de América la tasa de fertilidad es de 22.3; 13 para Canadá; 7 para Francia; y 5 para Alemania. Las mujeres adolescentes mexicanas representan el $16.5 \%$ del total de mujeres en edad fértil, siendo el grupo más grande con relación al total de mujeres en edad fértil (Consejo Nacional de Población [CONAPO], 2016). Lo anterior se relaciona con altas tasas de embarazo adolescente, un problema de salud pública que incrementa el riesgo de morbimortalidad materno-perinatal y conduce a una mayor vulnerabilidad social debido al rezago educativo, la asunción de roles de género tradicionales y desventajas laborales que perpetúan el ciclo intergeneracional de pobreza (Fondo de Población de las Naciones Unidas México, 2018). Para el 2017, el 17.9 \% de los embarazos en México se registraron en mujeres menores de 20 años (INEGI, 2018b). Dichas cifras sitúan a México como uno de los países con mayor tasa de fecundidad en la adolescencia entre los estados miembros de la OCDE (2019).

En lo que concierne a las ITS, cada día, un millón de personas en el mundo adquiere una ITS (WHO, 2019). En Estados Unidos de América, cerca de la mitad de los casos de ITS se dan en personas de 15 a 24 años. En México, del total de casos detectados del VIH, el $94.8 \%$ adquirieron el virus por vía sexual. Aunque las tasas de nuevos casos de VIH entre adolescentes de 10 a 19 años son relativamente bajas (2.3\%), en comparación con otros grupos etarios como el de 20 a 29 años $(28.5 \%)$-aproximadamente 12 veces mayor- (Centro Nacional para la Prevención y el Control del VIH y el sida [Censida], 2019), autores como de Jesús-Reyes y Menkes-Bancet (2017), consideran que en la adolescencia es cuando se adquiere la infección por VIH, pero su detección acontece en períodos posteriores de la vida. Esto evidencia la importancia de la prevención de ITS a temprana edad.

Uno de los elementos centrales para prevenir la adquisición de ITS y el embarazo en la adolescencia es el uso del condón masculino (Widman, Noar, Choukas-Bradley \& Francis, 2014). Sin embargo, su uso puede estar condicionado por elementos a nivel individual y social, entre ellos: el sexo, siendo los hombres quienes, por lo general, reportan mayor frecuencia de uso; el desconocimiento en la técnica de colocación; creencias; actitudes; estigma; falta de habilidades 
para negociar su uso; o el nivel socio económico (de Jesús-Reyes \& Menkes-Bancet, 2014; Kalamar, Bayer \& Hindin, 2016; Protogerou, Hagger \& Johnson, 2017).

La última Encuesta Nacional de Salud en México (Gutiérrez et al., 2012), mostró que alrededor del $15 \%$ de los adolescentes encuestados reportaron no saber que el condón se utiliza una sola vez, y el $24 \%$ contestó no saber si el condón puede ser útil para prevenir el embarazo y las ITS. Por su parte, se ha encontrado que el nivel socioeconómico está ligado a elementos socioestructurales, como el acceso a servicios de salud sexual y reproductiva; quienes menos acceso tienen a condones e información sexual son las personas con niveles económicos menos favorecidos (Gómez-Inclán \& Durán-Arenas, 2017; Wang, Lurie, Govindasamy \& Mathews, 2018).

La Estrategia Mundial para la Salud de la Mujer, el Niño y los Adolescentes (United Nations, 2016), que nace en el marco de la agenda de los Objetivos de Desarrollo Sostenibles, y la Estrategia del Sector Global de Salud en Infecciones de Transmisión Sexual 2016-2021 (WHO, 2016), hacen un llamado urgente a la adaptación y adopción de intervenciones integrales basadas en evidencia para salvaguardar la salud sexual y reproductiva de los adolescentes, así como al aprovechamiento del campo educativo para su implementación. Frente a la creciente problemática que significa el embarazo en la adolescencia y las ITS para la salud pública, y el impacto sobre las condiciones sociales de los adolescentes, el gobierno mexicano ha desplegado una serie de estrategias para hacer frente a dichas situaciones, entre la que se encuentra la Estrategia Nacional para la Prevención del Embarazo Adolescente (ENAPEA, Gobierno de la República, 2016). Dicha estrategia plantea como objetivo principal reducir la tasa de fertilidad al $50 \%$ en mujeres de entre 15 y 19 años, así como eliminar los casos de embarazo en mujeres menores de 14 años, para el 2030. Dentro del cuerpo estratégico, se recomienda el desarrollo e implementación de intervenciones, tanto en el contexto clínico como en todos aquellos donde se pueda captar adolescentes (por ejemplo, el educativo).

Las intervenciones cognitivo-conductuales surgen, frente a este panorama, como una herramienta positiva para prevenir los resultados adversos relacionados con la salud sexual y reproductiva de los adolescentes. Revisiones sistemáticas previas sobre intervenciones para la prevención de ITS y anticoncepción indican que las intervenciones, para ser efectivas sobre la variable resultado, necesitan de dos aspectos principales: 1) estar basadas en evidencia; y 2) teoría (Kalamar et al., 2016; Lopez, Grey, Chen, Tolley \& Stockton, 2016). De acuerdo con una de las 

percibida del uso del condón masculino en adolescentes mexicanos

revisiones sistemáticas más recientes para identificar intervenciones para anticoncepción efectivas de Cochrane (Lopez et al., 2016) y el banco de intervenciones disponibles por el CDC (Center for Disease Control and Prevention (CDC), s.f.)., una de las teorías que mejor efecto tiene sobre el uso del condón y anticoncepción en adolescentes es la Teoría Social Cognitiva (TSC) (Bandura, 1978; Bandura \& National Institute of Mental Health, 1986).

La TSC explica que la autoeficacia percibida (AE) responde a la confianza que el individuo tiene para lograr con éxito determinada conducta, por ejemplo: el uso del condón. Aquí lo que la persona sabe, o lo que ha hecho previamente por lo general no es el mejor predictor de su comportamiento subsecuente, dado que las creencias que posee sobre sus capacidades tienen una influencia importante sobre la forma en que puede llegar a comportarse. El proceso la AE implica que las personas se comprometan con una conducta, para después interpretar los resultados de sus acciones y con estos resultados se desarrollan creencias sobre si será capaz o no de llevar a cabo la conducta en posteriormente en contextos similares (Fig. 1).

Fig. 1. Proceso de AE aplicado al uso del condón.

Fig. 1. Self-efficacy (SE) process applied to condom use.

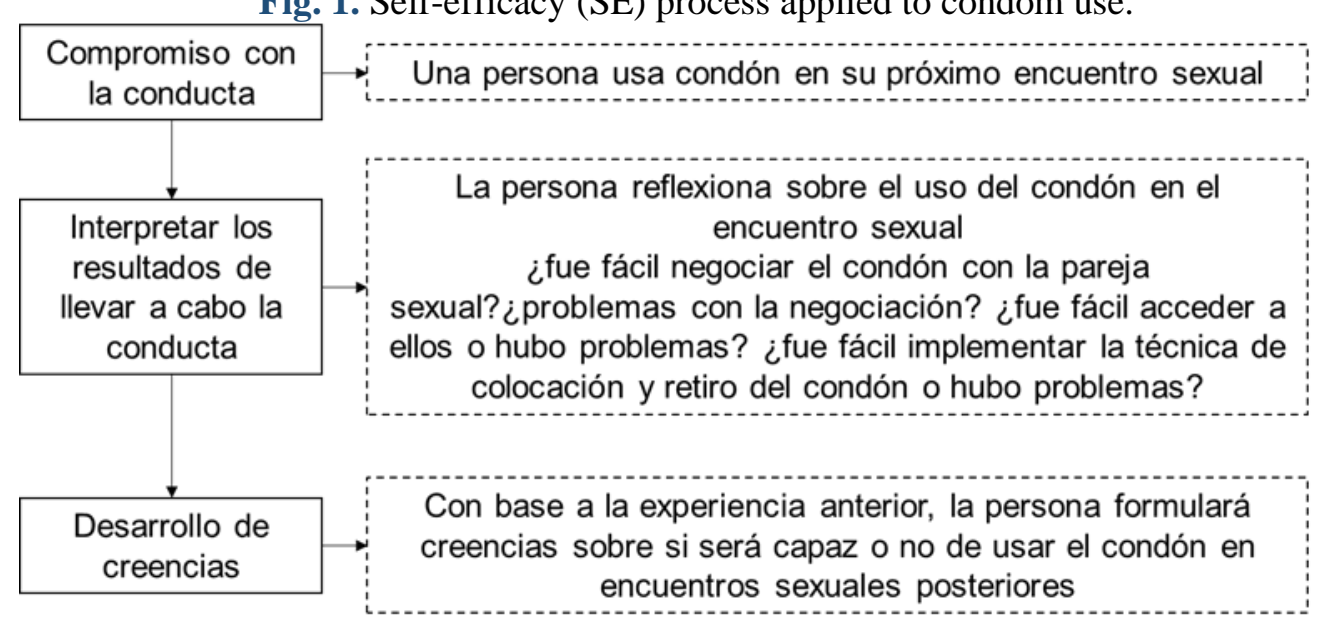

Fuente: Elaboración propia, con información de la TSC (Bandura, 1978; Bandura \& National Institute of Mental Health, 1986).

Source: Own elaboration, based on information from the SCT (Bandura, 1978; Bandura \& National Institute of Mental Health, 1986)

La AE se construye de cuatro elementos: 1) experiencias vicarias; 2) persuasión verbal o social; 3) experiencias anteriores; y 4) estados fisiológicos. Las experiencias vicarias se basan en experiencias observadas en otros, puede resultar muy enriquecedora cuando la persona no ha tenido la experiencia o conocimiento previo sobre la conducta. Del aprendizaje que adquiere un grupo, el 
resultado positivo que tenga uno de los sujetos puede incidir para que otro de ellos lo adopte como modelo a seguir. Por ejemplo, una demostración de la técnica del uso del condón a una persona que no ha tenido experiencias anteriores con el uso de este puede motivarle a aplicar la técnica en un encuentro sexual. La persuasión verbal o social, involucra la exposición a los juicios verbales de los demás; puede jugar una parte importante en el desarrollo de las creencias del adolescente sobre llevar a cabo la conducta. Las experiencias anteriores, son el resultado de la experiencia previa llevando a cabo la conducta. Por último, los estados fisiológicos se refieren a los estados anímicos, los cuales pueden influir sobre sus propias creencias para llevar a cabo la conducta. En la actualidad existen diversas intervenciones para promover la autoeficacia del uso del condón masculino, basadas en evidencia y teoría (Center for Disease Control and Prevention (CDC), s/f). Sin embargo, su implementación en contextos diferentes al original requiere de procesos de adaptación. El proceso de adaptación es importante, en la medida en que asegura que la intervención sea culturalmente adecuada para la población y el contexto de destino, respetando los elementos centrales de la misma (Rogers, 2003). Recientemente, Morales et al. (2019), adoptaron una intervención española para promover la salud sexual en adolescentes en Colombia, siguiendo un proceso sistemático de adaptación y obteniendo resultados favorables.

La adaptación de innovaciones es una etapa importante en los procesos de transferencia del conocimiento, ya que permite trasladar las innovaciones, estrategias e intervenciones basadas en evidencia que han sido previamente probadas y con resultados positivos a nuevos contextos (Straus, Tetroe \& Graham, 2009). El éxito de la adaptación de intervenciones requiere apegarse a modelos que han garantizado previamente la sistematización y rigurosidad del proceso y que permite replicar adaptaciones subsecuentes (McKleroy et al., 2006). Por ejemplo, de las intervenciones que se han adaptado a otros contextos exitosamente se encuentran "COMPAS” y "¡Cuídate!" (Villarruel, Jemmott \& Jemmott, 2006) originalmente diseñadas para adolescentes. La primera fue adaptada en Colombia y España (Espada, Escribano, Morales \& Orgilés, 2017; Morales et al., 2019), y la segunda a población mexicana (Gallegos, Villarruel, Loveland-Cherry, Ronis \& Zhou, 2008), ambas mostraron efectos significativos después de la adaptación.

Bajo estos preceptos, el objetivo principal de este estudio fue realizar la adaptación, evaluación de la aceptabilidad y el pilotaje de una intervención educativa basada en teoría y evidencia, la cual objetiva el incremento de la autoeficacia percibida del uso del condón masculino en adolescentes mexicanos. Se utiliza el "Mapa del Proceso de Adaptación: Un Enfoque 
Sistemático para Adaptar las Intervenciones Conductuales Basadas en Evidencia" (McKleroy et al., 2006).

\section{Método}

\section{Marco de referencia para la adaptación de la intervención}

La adaptación es la medida en que una innovación, en este caso una intervención, es modificada en el proceso de adopción e implementación (Rogers, 2003). La etapa de adaptación pretende transformar la innovación mediante la inclusión, eliminación o modificación de elementos existentes, cambiar las técnicas, actividades, dosis de entrega o realizar cambios culturales necesarios para la población de destino (McKleroy et al., 2006). Por lo general, una adaptación se considera necesaria para hacer modificaciones importantes para una población en particular, de acuerdo con sus necesidades, lo que podría perfeccionar la intervención con respecto al resultado esperado, sin perder de vista los elementos centrales de la intervención (Kelly et al., 2000). De acuerdo con lo anterior, se determinaron algunos motivos por los cuales se requirió efectuar una adaptación: la primera fue simplificar la innovación, aplicar a otra población, resumir sesiones y realizar cambios por el tipo de población.

El modelo de McKleroy et al. (2006), es una herramienta para la adaptación de intervenciones del VIH, y se compone de 3 etapas y 5 pasos. El primer paso se refiere a la valoración: requiere de conocer las características de la población de interés, así como la revisión de la intervención. El segundo paso es sobre la selección: se decide qué elementos de la intervención original requieren transformarse. El tercer paso radica en la preparación de la intervención: incluye organizar actividades, modificar los materiales y realizar pruebas a la población de interés. El cuarto paso es llevar a cabo una prueba piloto del plan de implementación

y revisar sus elementos. El quinto paso consiste en la implementación de la intervención adaptada: se realiza una revisión profunda de ésta y se efectúan modificaciones menores necesarias (Fig. 2). 
Fig. 2. Proceso de adaptación de intervenciones.

Fig. 2. Adaptation process of the intervention.

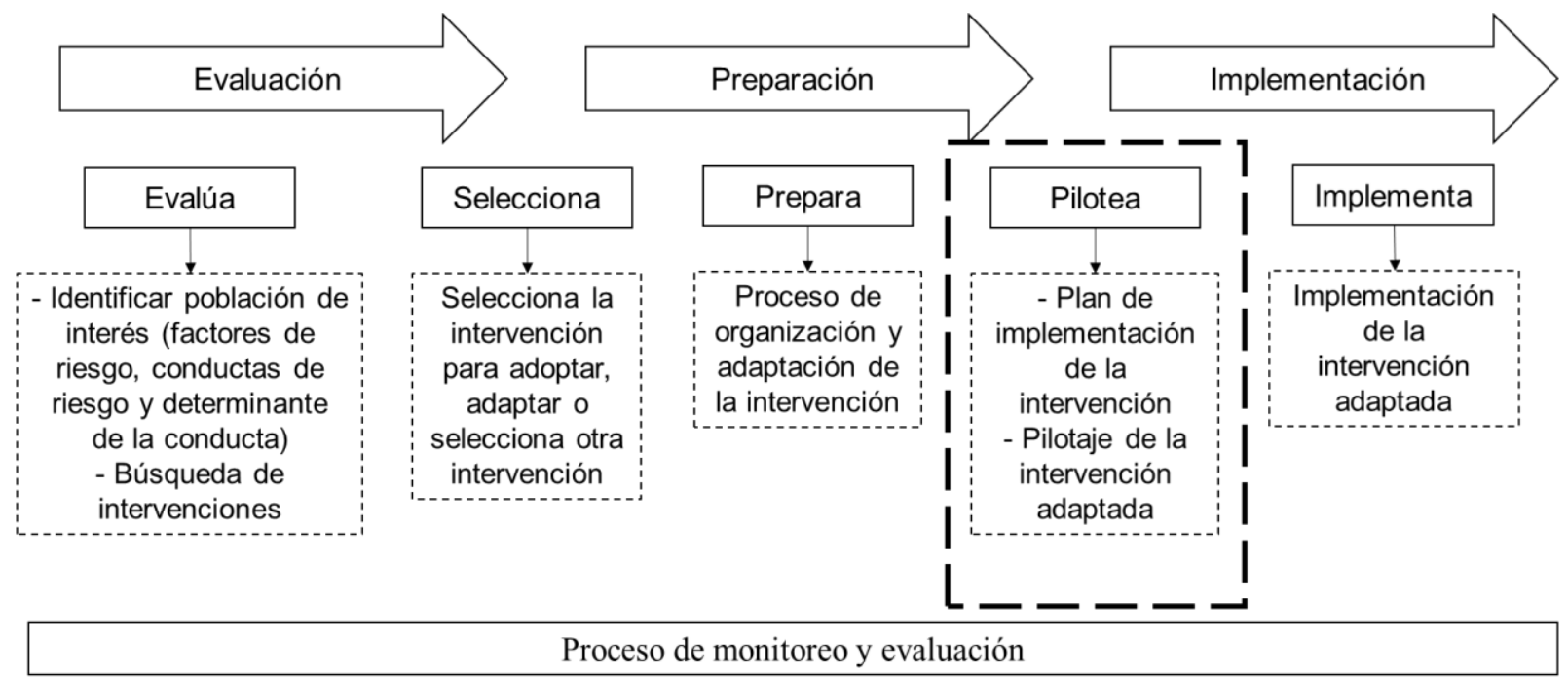

Fuente: Elaboración propia, con base en el "Mapa del Proceso de Adaptación: Un Enfoque

Sistemático para Adaptar las Intervenciones Conductuales Basadas en Evidencia” (McKleroy et al., 2006)

Source: Own elaboration, based on the "Map of the Adaptation Process: A Systematic Approach to Adapt Evidence-Based Behavioral Interventions” (McKleroy et al., 2006).

\section{Escenario}

Puebla es uno de los 32 estados que conforman los Estados Unidos Mexicanos, con una población de alrededor de 6, 183, 320 habitantes, distribuidos en los 217 municipios que le componen. El grado promedio de escolaridad es de 15 años. Es uno de los estados con mayor población indígena (INEGI, 2015a) y alrededor del $74 \%$ de la población tiene adscripción católica. Puebla se encuentra entre los primeros 10 estados en México con mayor número de embarazo en mujeres menores de 20 años (INEGI, 2015c), donde alrededor del $18.8 \%$ de los embarazos totales se registran en este grupo (INEGI, 2015b).

\section{Participantes}

Se reclutaron adolescentes pertenecientes a una preparatoria adscrita a una universidad de Puebla. Se incluyeron en el estudio solo a aquellos que no hubieran estado expuestos a algún tipo de plática, taller o sesión relacionada con el uso del condón o sobre salud sexual y reproductiva en los últimos 12 meses. Quince días antes de la aplicación de la intervención, se acudió a la institución para invitar a posibles participantes. En caso de que fueran menores de edad, se les entregó el consentimiento informado para sus tutores, mismo que se les requirió para ser parte de la 
intervención, junto con el propio consentimiento informado de los participantes. El estudio estuvo apegado a la Ley General de Salud en Materia de Investigación para la Salud (Secretaría de Salud, 1983) y fue aprobado por el Comité de Investigación de la Facultad de Enfermería de la Benemérita Universidad Autónoma de Puebla (Número de Registro: FE/SIEP/1560/2016).

\section{Procedimiento}

Valoración. Esta adaptación se realizó para implementarse en adolescentes de preparatoria de la ciudad de Puebla, Puebla. Se realizó una búsqueda exhaustiva de la literatura con el fin de identificar intervenciones dirigidas a incrementar la autoeficacia del uso del condón masculino. Las intervenciones debían tener como criterio básico estar basadas en teoría, utilizando el constructo de AE. Una vez identificadas las intervenciones, se procedió a contactar a los autores de los artículos para solicitar los manuales de implementación de las intervenciones. Se hizo de conocimiento de los autores los objetivos de la obtención de los manuales: 1) evaluarlos para conocer la factibilidad de adaptarlos a población mexicana; 2) si fuera así, traducirlos al español; 3) hacer modificaciones; y 4) implementarlos. Se mantuvieron entrevistas con los autores para indagar o esclarecer dudas con respecto a los elementos centrales de la intervención, la viabilidad de adaptarlo al escenario, los participantes y las dosis distintas. También se buscó compartir experiencias sobre la implementación.

Selección. Descripción de la intervención "Usando Condón". Se seleccionaron dos intervenciones para adaptar en una sola. La primera intervención denominada "The Positive Choices Mapping" (PCM), cuyo objetivo es aumentar la AE del uso del condón masculino mediante 6 sesiones de 60 minutos y se basa en la TSC, se implementó en población urbanaafroamericana (Czuchry, Timpson, Williams, Bowen \& Ratliff, 2009). La segunda intervención llamada "Condom Promotion" se desarrolló en mujeres universitarias de Estados Unidos de América, consta de una sola sesión de 45 minutos que tiene como objetivo incrementar el uso del condón masculino, así como los beneficios percibidos, las actitudes positivas hacia él, la aceptación percibida de la sexualidad, el control sobre el contexto sexual, la AE percibida del condón e incrementar las intenciones de usar condón (Bryan, Aiken \& West, 1996; material suplementario).

Se estableció el modo de entrega en dos sesiones presenciales con una duración de 90 minutos cada una, con múltiples actividades dentro del aula para practicar la comunicación sexual 
con parejas sexuales mediante ejemplos hipotéticos, así como el modelaje del uso del condón. El acortamiento del número de sesiones se hizo con base a la identificación de los elementos centrales de las intervenciones, los cuales estaban descritos en los artículos publicados de cada uno de ellos, así como a través de las entrevistas que se llevaron a cabo con los autores.

Es importante mencionar que los elementos centrales de las intervenciones, de acuerdo con los lineamientos de adaptación, no deben eliminarse, pero las actividades para abordarlos sí pueden sufrir modificaciones. Por ejemplo, uno de los elementos centrales de "The PCM" es la negociación del uso del condón masculino en personas que ya viven con el virus, sin embargo, para la población de estudio, aunque también se incluye la negociación del condón, el objetivo cambia. En el primer caso la negociación del uso del condón objetiva evitar la reinfección o superinfección del VIH y en el caso actual evitar la adquisición del VIH por primera vez, por lo que los escenarios utilizados en las actividades cambiaron. Una vez identificados los elementos centrales y eliminados aquellos no centrales y que no eran de interés para la población de estudio actual (por ejemplo: actividades para personas que vivían con el VIH) se organizó la información y actividades quedando dos sesiones (material suplementario).

Dado que las intervenciones que sirvieron de base para la adaptación estaban fundamentadas en la AE, en el proceso de adaptación se cuidó que los principios de la teoría no se vieran alterados. Lo anterior, se logró mediante un escrutinio constante y continuo de las actividades y contenido para corroborar que correspondían a cada parte de la AE (persuasión verbal, experiencias anteriores etc.) con ayuda de una experta en intervenciones conductuales en adolescentes y salud sexual y reproductiva.

Otro ejemplo es la estrategia del mapeo cognitivo, central en la intervención PCM, que se respetó y se integró en la intervención "Usando Condón”. El mapeo cognitivo ayuda a crear y explorar "representaciones cognitivas que apoyan o impiden el cambio conductual haciendo los pensamientos concretos y visibles" (Czuchry et al., 2009). El mapeo puede ayudar a los participantes a mejorar la autoeficacia, porque permite plasmar y contraponer gráficamente pensamientos/creencias y hechos reales sobre determinado tema (por ejemplo: el uso del condón y el placer). En el artículo original de Czuchry et al. (2009), se pueden observar algunos ejemplos del mapeo cognitivo.

La estructura de la intervención incluyó metas, objetivos, estrategias y métodos, materiales requeridos, tiempo requerido, justificación, procedimiento y notas para facilitador. Además de las 
Adaptación, aceptabilidad y pilotaje de "Usando Condón". Una intervención para incrementar la autoeficacia percibida del uso del condón masculino en adolescentes mexicanos

actividades a desarrollar por los participantes (Tablas 1 y 2). La intervención se evalúa mediante una prueba test y re-test, es decir, se realiza una medición antes y después de la exposición de la intervención, mediante un instrumento que mide la autoeficacia para el uso del condón en adolescentes. Al final de la intervención, con el objetivo de evaluar la aceptabilidad de la intervención y seguir mejorando su estructura, se les pide a los participantes que escriban en una hoja blanca lo que piensan de la intervención (contenido, actividades, herramientas, facilitadora) y cómo se sintieron.

Tabla 1. Contenido y actividades de la primera sesión de "Usando Condón".

Table 1. Content and activities of session one of "Usando Condón".

\begin{tabular}{|c|c|}
\hline \multicolumn{2}{|r|}{ Primera Sesión } \\
\hline Contenido Temático & Actividad \\
\hline $\begin{array}{l}\text { VIH/Sida (creencias, } \\
\text { medios de } \\
\text { transmisión) }\end{array}$ & $\begin{array}{l}\text { - MC creencias de transmisión del VIH. } \\
\text { - Memorama con imágenes de posibles vías de } \\
\text { transmisión/adquisición, que el participante organiza conforme el } \\
\text { cree que son rutas de transmisión o no. } \\
\text { - Carteles con datos estadísticos y vías de transmisión que marca la } \\
\text { evidencia. }\end{array}$ \\
\hline ITS & $\begin{array}{l}\text { - MC para conocer cuáles son las ITS que conocen. } \\
\text { - Carteles basados en evidencia para mostrar información sobre ITS } \\
\text { (prevalencia, signos y síntomas, repercusiones). }\end{array}$ \\
\hline $\begin{array}{l}\text { Embarazo en la } \\
\text { adolescencia }\end{array}$ & $\begin{array}{l}\text { - MC de consecuencias percibidas del embarazo en la adolescencia. } \\
\text { - Carteles basados en evidencia de las consecuencias del embarazo } \\
\text { en la adolescencia. }\end{array}$ \\
\hline Sexo seguro & $\begin{array}{l}\text { - MC ¿qué es el sexo seguro? ¿por qué los adolescentes usan o no el } \\
\text { condón? ¿cómo se sentirían al negociar el uso del condón con } \\
\text { parejas sexuales ya establecidas? } \\
\text { - Diálogos ficticios basados en evidencia donde adolescentes } \\
\text { mencionan porque no utilizan condón. } \\
\text { - Escenarios imaginarios para la compra de condones. } \\
\text { - Se les pide traer un condón en su bolsa o mochila durante los días } \\
\text { entre la primera y segunda intervención. } \\
\text { - En parejas practican diálogos de negociación del uso del condón. }\end{array}$ \\
\hline $\begin{array}{l}\text { Derechos sexuales y } \\
\text { necesidad de intimidad }\end{array}$ & $\begin{array}{l}\text { - MC creencias sobre derechos sexuales y qué es la intimidad. } \\
\text { - Carteles basados en evidencia sobre derechos sexuales de las y los } \\
\text { adolescentes. }\end{array}$ \\
\hline
\end{tabular}

Fuente: Elaboración propia. Intervención "Usando Condón" derechos de autora. Source: Own elaboration. "Usando Condón” intervention author copyright. 
Tabla 2. Contenido y actividades de la segunda sesión de "Usando Condón".

Table 2. Content and activities of session two of "Usando Condón"

\begin{tabular}{|c|c|}
\hline \multicolumn{2}{|r|}{ Segunda Sesión } \\
\hline Contenido Temático & Actividad \\
\hline $\begin{array}{l}\text { Recordatorio de la } \\
\text { sesión anterior }\end{array}$ & - Lluvia de ideas. \\
\hline $\begin{array}{l}\text { Roles de género y } \\
\text { expectativas de una } \\
\text { relación }\end{array}$ & - MC roles de género y que se espera de una relación sexual. \\
\hline Uso del condón & $\begin{array}{l}\text { - } \quad \text { MC condón (creencias y precauciones al usarlo). } \\
\text { - Se proporcionan } 3 \text { condones (estirar, oler, sentir consistencia). } \\
\text { - } \quad \text { Póster pasos correctos del uso del condón. } \\
\text { - Facilitadora muestra la técnica del correcta e incorrecta del } \\
\text { uso del condón con un modelo anatómico de pene. } \\
\text { - En grupos los participantes replican el modelaje del uso del } \\
\text { condón con modelos anatómicos de pene, con retroalimentación } \\
\text { de sus pares. }\end{array}$ \\
\hline $\begin{array}{l}\text { Alternativas del } \\
\text { condón }\end{array}$ & $\begin{array}{l}\text { - Se muestran condones de diferentes tamaños, formas, colores } \\
\text { y olores. } \\
\text { - Se les proporcionan los distintos tipos de condones que } \\
\text { entregan las diferentes instituciones de salud pública. } \\
\text { - Se entregan condones de diferentes colores, sabores, tipo de } \\
\text { lubricante y texturizado (se aclara que estos solo son accesibles en } \\
\text { farmacias o comercios privados). }\end{array}$ \\
\hline $\begin{array}{l}\text { Pastilla del día } \\
\text { siguiente }\end{array}$ & $\begin{array}{l}\text { - MC qué se hace cuando el condón falla. } \\
\text { - Cartel basado en evidencia con información sobre la pastilla } \\
\text { del día siguiente. }\end{array}$ \\
\hline $\begin{array}{l}\text { Conclusión de las } \\
\text { sesiones }\end{array}$ & $\begin{array}{l}\text { - Resumen mediante lluvia de ideas del contenido de las } \\
\text { sesiones. }\end{array}$ \\
\hline
\end{tabular}

Fuente: Elaboración propia. Intervención "Usando Condón" derechos de autora.

Source: Own elaboration. "Usando Condón" intervention author copyright.

Preparación. Mediante la valoración de las intervenciones, se decidió modificar aspectos lingüísticos (lenguaje coloquial mexicano), aspectos culturales (por ejemplo, en la actividad de roles de género, escenarios de negociación del uso del condón). La facilitadora de la intervención recibió orientación sobre implementación de intervenciones cognitivo-conductuales en adolescentes sobre el uso del condón y prevención de ITS, durante un mes y medio. La asistente de la facilitadora recibió entrenamiento por parte de la facilitadora para entregar el material a los 
participantes sin obstruir la entrega de la intervención. El manual y material de la intervención fue retroalimentado por una experta en intervenciones de prevención de ITS en adolescentes.

La preparación también incluyó organizar el acceso a los posibles participantes, lo cual requirió la gestión administrativa en una preparatoria adscrita a una universidad pública de Puebla. Se entregaron oficios para solicitar el campo con un mes de anticipación y las autoridades institucionales solicitaron una entrevista para solicitar información sobre el contenido y aspectos éticos (consentimiento de los padres específicamente).

Prueba piloto de la adaptación. Se planteó una prueba piloto para evaluar las modificaciones, la aceptabilidad y fidelidad de la intervención "Usando Condón”, para conocer qué partes requerían adaptarse de nueva cuenta o esclarecerlas, así como registrar los tiempos programados para cada actividad para su reajuste si fuese necesario. A la prueba piloto se integraron adolescentes de forma voluntaria. Se les entregó el consentimiento informado para sus padres una semana antes de la intervención y solo aquellas personas que lo entregaron firmado, así como su asentimiento informado, participaron en la prueba piloto.

\section{Mediciones y recolección de la información}

Aceptabilidad. La aceptabilidad de la intervención se evalúo mediante la asistencia y deserción en las sesiones. La satisfacción de la intervención se evaluó al final de la última sesión, mediante hojas en blanco en donde los participantes plasmaron sus opiniones de forma anónima, sobre la intervención (por ejemplo: contenido, facilitadora). Para el análisis de los discursos se utilizó análisis temático (Braun \& Clarke, 2006) auxiliado por el paquete de análisis de datos cualitativos Nvivo 11 Plus para Windows.

Fidelidad de implementación. La intervención fue aplicada por una sola facilitadora con licenciatura en enfermería, la cual se apegó en todo momento al manual de la intervención "Usando Condón", palomeando cada actividad y contenido entregado. Asimismo, recibió orientación durante un mes y medio en intervenciones cognitivo-conductuales.

Autoeficacia percibida para el uso del condón masculino. Para medir la variable resultado de la intervención "Usando Condón”, se utilizó la Escala de Autoeficacia para el Uso del Condón en 
Adolescentes Mexicanos con Alfa de Cronbach de .086 (Carrera Huerta et al., 2016). Se trata de un instrumento escrito en castellano, adecuado con un lenguaje coloquial para que el adolescente mexicano lo entienda de una manera más simple. Se diseñó para medir el nivel de AE para el uso del condón, y consta de 17 ítems. El patrón de respuesta para cada reactivo incluye una escala tipo Likert de cinco opciones, cada una corresponde a una respuesta análoga visual, representada por “caritas": 1) nunca; 2) casi nunca; 3) algunas veces; 4) casi siempre; y 5) siempre. Cada respuesta se suma, obteniendo un puntaje mínimo de 17 y un máximo de 85, en donde a mayor puntaje, mayor AE sobre el uso correcto del condón. La escala se aplicó antes de empezar la intervención (pre-prueba) y después de finalizar la intervención (post-prueba), para analizar el cambio de la variable de autoeficacia percibida del uso del condón después de la exposición a la intervención

Análisis estadístico. Los datos se procesaron a través del Stadistical Package for the Social Sciences (SPSS versión 21). Con el objetivo de describir las características de la muestra y las variables de interés, se utilizaron medidas de tendencia central, así como porcentajes y frecuencias. Los datos obtenidos de la Escala de Autoeficacia en el Uso del Condón en Adolescentes Mexicanos se convirtieron a índices de 0 a 100. Los índices se sometieron a la prueba de Kolmogorov Smirnov indicada para muestras mayores a 50 sujetos, para determinar la curva de la distribución de las variables $(p>.05)$, y se utilizó la prueba paramétrica $t$ de Student para muestras relacionadas para medir las variaciones entre las mediciones de prueba (medición 1 [M1]) y post-prueba (medición 1 [M2]) correspondientes a la evaluación de la intervención.

\section{$\underline{\text { Resultados }}$}

Se reclutaron 61 adolescentes de los cuales se incluyeron 56. Los participantes se excluyeron de la intervención porque no entregaron consentimiento informado de los padres (2) y porque habían acudido a una plática relacionada al uso del condón en los últimos 12 meses. La edad media fue de 16.98 años; participaron 33 adolescentes de sexo femenino y 23 de masculino (Tabla 4).

\section{Adaptaciones a la intervención "Usando Condón"}

Se adoptaron los componentes esenciales de las intervenciones tales como lo referente a la información y actividades sobre VIH/sida, ITS, las actividades para fomentar el uso del condón (creencias de reducción de placer, negociación, acceso, beneficios, consecuencias de no usarlo 
[embarazo, ITS], barreras), modelaje del condón a través de modelos anatómicos de pene e imaginarios de los roles de género sobre sexualidad. De las técnicas didácticas para implementar la intervención se utilizó el MC, los ejemplos de historias, participación de la audiencia y el modelaje del uso del condón con modelos anatómicos de pene. Se integraron actividades con diálogos para practicar la negociación del uso del condón. Los datos utilizados sobre prevalencias de ITS se modificaron para adolescentes. Asimismo, se extendió la información referente al embarazo como consecuencia, con datos basados en evidencia específicos sobre adolescentes. Se integró contenido sobre la anticoncepción de emergencia por recomendación de la guía de práctica clínica sobre uso del condón (Secretaría de Salud, 2011).

Tabla 3. Características demográficas de los participantes de "Usando Condón". Table 3. Participants' demographic data of "Usando Condón".

\begin{tabular}{|cc|}
\hline & Grupo pilotaje $(\mathbf{N}=\mathbf{5 6})$ \\
\cline { 2 - 2 } Edad media [DE] & \multicolumn{1}{c|}{$\mathbf{1 6 . 9 8 \pm . 3 5 6}$} \\
\hline Sexo & $33(58.9 \%)$ \\
Hujeres & $23(41.1 \%)$ \\
\hline Viviendo con & \\
Padres & $54(96.4 \%)$ \\
Familiares & $2(3.6 \%)$ \\
& \\
\hline Religión & $30(53.6 \%)$ \\
Católica & $7(12.5 \%)$ \\
Cristiana & $13(23.2 \%)$ \\
Ninguna & $6(10.8 \%)$ \\
Otra &
\end{tabular}

Fuente: Elaboración propia con datos demográficos del pilotaje de "Usando Condón". Source: Own elaboration based on demographic data of the pilot testing of "Usando Condón".

Se eliminaron las actividades y temas referentes a la develación del seroestado de VIH y negociación del uso del condón con base en el seroestado de VIH, en el caso de la intervención de (Czuchry et al., 2009). Se modificaron las actividades referentes a la aceptación de la sexualidad para ser aplicables tanto para hombres como para mujeres (Bryan et al., 1996). Los escenarios y 
dosis de ambas intervenciones se modificaron, integrando el contenido de las seis sesiones de "The Positive Choice Mapping” y el contenido de la sesión única de "Condom Promotion", en dos sesiones de 90 minutos. La facilitadora en el caso de "Usando Condón", fue una enfermera, a diferencia de las otras intervenciones implementadas por psicólogos. El material suplementario muestra una tabla comparativa de las características de las intervenciones utilizadas para la adaptación (componente, dosis, duración, facilitador, contenido temático) y en la columna final lo referente a la intervención "Usando Condón". En la tabla se puede observar cuáles fueron los elementos eliminados y modificados.

Con base a las sugerencias de los participantes se incluyeron en el manual de la intervención (para la fase final del modelo de adaptación): para la primera sesión, actividades para hacer más interactiva y participativa la primera sesión. Se incluyó más información hacia la temática de ITS, específicamente sobre el VPH, herpes, sífilis y gonorrea. La información, que anteriormente se entregaba únicamente mediante carteles, se combinó con diapositivas, cuidando siempre que no estuvieron cargadas de texto y que fueron visiblemente atractivas. La sesión dos no requirió de ningún tipo de adaptación. El único aspecto que no pudo integrarse a la intervención fue el testimonio de adolescentes que vivieran con el VIH.

\section{Aceptabilidad}

La asistencia a la intervención fue del 100 \%. En general, los discursos de retroalimentación de la intervención fueron positivos:

1) "Me parecieron muy buenas las pláticas por las diferentes actividades que se realizaron en estas 2 sesiones. La actividad de poner el condón a un "pene" fue algo bueno porque generalmente nos dan los pasos, pero no lo llevamos a la práctica y me parece que lo que se fomentó en estas pláticas fue más práctico que teórico. Me agrado demasiado".

2) "Las pláticas me parecieron interesantes, además fueron muy dinámicas, ya que anteriormente las pláticas que habíamos tenido sobre sexualidad eran demasiado monótonas y aburridas". 
3) "Me pareció muy interesante y explicativa. Nos aclararon muchas cosas que anteriormente nos habían explicado, pero nos dejaron con algunas dudas. Ahora nos enseñaron y aprendimos de forma interactiva sobre estas prácticas”.

Las críticas para fortalecer la intervención giraron en torno a cuatro temáticas: 1) material tecnológico de apoyo que se utilizó; 2) el contenido sobre ITS; 3) actividades prácticas vs. teóricas; y 4) la inclusión de testimonios de adolescentes viviendo con el VIH. Sobre el uso de recursos tecnológicos, algunos participantes mencionaron:

1) "No quitaría nada, todo me gustó ya que trajeron mucho material. Agregaría, quizá, que investigaran a la escuela que van [a desarrollar la intervención] si cuentan con proyector (cañón) y hacer presentaciones electrónicas, ya que puede ser más rápido y explícito, quizá entregar imágenes”.

2) "Creo que el tono de voz y dicción de la que dio la plática fueron los correctos, aunque sería mejor que utilizara más audiovisuales o diapositivas, ya que a mi parecer es más práctico, pero en cuanto al contenido siento que estuvo bien".

Se identificaron recomendaciones hacia el incremento de información sobre otras ITS además del VIH/sida: "Creo que la forma en que dan las pláticas está muy bien, aunque yo le agregaría más tiempo para hablar sobre las demás enfermedades y no solo del SIDA todo el tiempo". Se recomendó incluir cuestiones prácticas y actividades para hacer la primera sesión menos informativa y teórica: "Estas dos practicas se me hicieron interesantes, en especial esta última, porque fue más práctica. La primera sección estuvo bien, el problema fue que paso más lenta". Algunos participantes sugirieron la introducción de testimonios de adolescentes que vivieran con el VIH: "Y con respecto a lo del VIH les falta traer evidencias de personas que lo han padecido".

\section{Fidelidad de la Implementación}

Una sola facilitadora quién llevó a cabo la intervención. El palomeo de las actividades muestra que se completó la intervención en un $96 \%$. De los escenarios propuestos para la negociación del uso del condón solo se completaron 2 de los 3 propuestos y no se abordaron 2 de las 4 diapositivas referentes a datos estadísticos del VIH. 


\section{Medición preliminar sobre los efectos de la intervención}

La Escala de Autoeficacia para el Uso del Condón en Adolescentes mexicanos mostró índices confiables en ambas mediciones pre-prueba y post-prueba $(\alpha=.839, \alpha=0.759$ respectivamente). A nivel descriptivo, los resultados evidencian el incremento de la autoeficacia percibida del uso del condón entre la pre-prueba $(67.78 \pm 17.857)$ y la post-prueba $(89.31 \pm 9.085)(p>0.38)$. Por su parte, la prueba de $t$ de Student corrobora el cambio en la variable resultado $(21.532 \pm 19.043$; IC $95 \%-26.632,-16.433 ; p<.05)$.

\section{Discusión o conclusiones}

El objetivo de este estudio fue llevar a cabo la adaptación y pilotaje de una intervención educativa basada en teoría para incrementar la autoeficacia percibida del uso del condón masculino en una muestra de adolescentes mexicanos, para lo cual se siguió el modelo de adaptación de intervenciones de Mckleroy et al. (2006) hasta la etapa de pilotaje. "Usando Condón" se basó en dos intervenciones anteriores (Bryan et al., 1996; Czuchry et al., 2009) cuya estructura teórica se basó en el constructo de AE. Como parte del proceso de adaptación se hizo una revisión de la literatura exhaustiva con el fin de identificar intervenciones basadas en teoría que objetivaran elevar la AE para el uso del condón. Se eligieron aquellas que cumplieron con los criterios y cuyos manuales y permisos de adaptación fueron compartidos por los autores. Sin eliminar elementos centrales de las intervenciones, los cuales fueron previamente identificados por los autores originales, se modificó parte del contenido de las sesiones (lenguaje, actividades; McKleroy, 2006).

Las modificaciones a las intervenciones e integración de información competente a los adolescentes estuvieron basadas en evidencia. La intervención final estuvo compuesta de dos sesiones de 90 minutos cada una. El acortamiento de las sesiones se discutió con los autores originales de las sesiones y con una experta en el desarrollo de intervenciones sobre salud sexual y reproductiva en adolescentes, de tal forma que se aseguró que el contenido no alteraba los componentes centrales de las intervenciones base. Intervenciones previas de entre 1 a 2 sesiones han mostrado tener impactos significativos sobre el uso del condón, como la "Consejería Personalizada Cognitiva" (Personalized Cognitive Counselling; CDC, 2019) compuesta por una sesión para reducir los encuentros sexuales sin uso del condón. También las intervenciones de una sola sesión "Sin buscar excusas/No excuses" (CDC, 2019b) y "Sister to Sister" (CDC, 2019c) cuyo objetivo es incrementar la sexualidad segura (uso del condón, habilidades de negociación etc.) 

percibida del uso del condón masculino en adolescentes mexicanos

La intervención "Usando Condón" fue ampliamente aceptada, con recomendaciones menores hacia los métodos tecnológicos para mostrar la información referente al contenido de la información, aumento de la información sobre ITS y la inclusión de testimonios de adolescentes viviendo con el VIH, siendo este último el único que no pudo introducirse en el contenido de la intervención. Este dato podría hablar de la relevancia que tiene para los adolescentes conocer la experiencia de personas que les son similares (otros adolescentes), en vías de generar referentes de resultados de salud de los cuales se perciben en riesgo o no.

De forma preliminar se puede afirmar que "Usando Condón" es una intervención que logra incrementar los niveles de AE del uso del condón masculino en adolescentes, pero que necesita implementarse en una muestra más grande de adolescentes. La implementación final, sugerida por McKleroy et al. (2006), como el último paso para la adaptación necesita integrar las modificaciones derivadas de este pilotaje. La intervención puede ser utilizada por profesionales de salud en contextos similares. Quiénes sean los facilitadores de la intervención necesitan de previa capacitación en el ejercicio de intervenciones conductuales y más específicamente con adolescentes, ya que esta población tiene características específicas de aprendizaje e interacción social.

La adaptación de innovaciones y su implementación son estrategias de aplicación del conocimiento importantes para el proceso de transferencia del conocimiento. La transferencia del conocimiento es esencial para mejor la salud poblacional, los servicios de atención a la salud y los procesos de toma de decisiones a nivel estructural (Straus et al., 2009). Es necesario considerar que, aunque existen intervenciones basadas en evidencia y teoría que han mostrado efectos significativos sobre la variable resultado, éstas necesitan de procesos sistemáticos de adaptación cuando se desee trasladarlas a contextos y/o poblaciones distintas. Por ejemplo, el CDC (s/f) tiene a disposición virtual una gama de intervenciones efectivas que se caracterizan por estar basadas en evidencia y teoría. Con lo anterior se pretende que, al ofrecer información sobre dichas intervenciones, los manuales de implementación y herramientas de capacitación, éstas puedan trasladarse a otros contextos y poblaciones mediante procesos sistemáticos de adaptación/tropicalización.

Los resultados de estudio deben considerarse tomando en cuenta que se trata de un proceso de adaptación de intervenciones, se consideró una muestra pequeña a conveniencia por lo que en estudios posteriores se recomienda documentar las barreras/estrategias para acceder a muestras más 
grandes y negociación del acceso al campo. Asimismo, solo se evaluó la AE para el uso del condón masculino, variables como conocimiento sobre ITS no se midieron dado que el objetivo central era pilotear la intervención y medir la variable principal.

Se recomienda registrar sistemáticamente si se harán modificaciones a la intervención en caso de necesitarse para aplicar a otro contexto o población y considerar la evidencia para la toma de decisiones. Se sugiere considerar las limitantes socio-estructurales en las instituciones educativas, como la disposición de las instituciones para que este tipo de intervenciones sean implementadas y la disposición de los padres y madres de firmar el consentimiento informado para que los adolescentes menores de edad participen. Aquí valdría la pena desarrollar un análisis y propuesta para la reformulación de los lineamientos éticos en investigación, para que los adolescentes menores de edad puedan participar en este tipo de intervenciones sin el consentimiento de sus padres, respetando los derechos sexuales y reproductivos de los adolescentes en México (CONAPO, Secretaría de Gobernación, INMUJERES, 2016). En contextos como el de México, también es importante considerar la religión y el rol de los grupos conservadores en la interposición de barreras para la implementación de programas de Educación Sexual Integral (Chandra-Mouli, Gómez Garbero, Plesons, Lang \& Corona Vargas, 2018).

\section{Agradecimientos}

Agradecemos al Consejo Nacional de Ciencia y Tecnología (CONACYT), por la beca de posgrado (\#572000) otorgada para la primera autora y que posibilitó el desarrollo de la presente investigación. A las Maestras en Enfermería Guadalupe Antonio González y Natalia Montes de Oca Chávez, por su colaboración en la etapa de recolección. Y a la preparatoria que abrió sus puertas para la adaptación de la intervención.

\section{$\underline{\text { Referencias }}$}

Bandura, A. (1978). Self-efficacy: Toward a unifying theory of behavioral change. Advances in Behaviour Research and Therapy, 1(4), 139-161. DOI: https://doi.org/10.1016/0146$\underline{6402(78) 90002-4}$

Bandura, A. \& National Institute of Mental Health. (1986). Social foundations of thought and action: A social cognitive theory. Englewood Cliffs, NJ, US: Prentice-Hall, Inc.

Braun, V. \& Clarke, V. (2006). Using thematic analysis in psychology. Qualitative Research in 
Psychology, 3(2), 77-101. DOI: 10.1191/1478088706qp063oa

Bryan, A. D., Aiken, L. S. \& West, S. G. (1996). Increasing condom use: Evaluation of a theorybased intervention to prevent sexually transmitted diseases in young women. Health Psychology, 15(5), 371-382. DOI: 10.1037//0278-6133.15.5.371

Carrera Huerta, S., Soto Carrasco, A. A., Muñoz, V. P., Sánchez Salinas, R., Osorio Ramírez, J. L., Abeille Mora, E. \& Landeros Olvera, E. (2016). Construcción y validación de la escala de autoeficacia para el uso del condón en adolescentes mexicanos. Enfermería Neurológica, 15(2), 70-79. DOI: https://doi.org/10.37976/enfermeria.v15i2.228

Center for Disease Control and Prevention [CDC]. (s/f). Effective Interventions. HIV Prevention that works. Recuperado de https://effectiveinterventions.cdc.gov/

CDC. (2019a). Personalized Cognitive Counseling. Recuperado de https://effectiveinterventions.cdc.gov/en/hiv-testing/group-2/personalized-cognitivecounseling

CDC. (2019b). Sin Buscar Excusas/No Excuses. Recuperado de https://effectiveinterventions.cdc.gov/en/hiv-negative-persons/group-3/sin-buscar-excusas$\underline{\text { no-excuses }}$

CDC. (2019c). Sister to Sister. Recuperado de https://effectiveinterventions.cdc.gov/en/hivnegative-persons/group-3/sister-to-sister

Centro Nacional para la Prevención y el Control del VIH y el sida [Censida]. (2019). Vigilancia Epidemiológica de casos de VIH/SIDA en México Registro Nacional de Casos de SIDA Actualización al ler. trimestre del 2019. Recuperado de https://www.gob.mx/cms/uploads/attachment/file/468354/RN_1erTrim_2019.pdf

Chandra-Mouli, V., Gómez Garbero, L., Plesons, M., Lang, I., \& Corona Vargas, E. (2018). Evolution and Resistance to Sexuality Education in Mexico. Global health, science, and practice, 6(1), 137-149. Recuperado de http://www.ncbi.nlm.nih.gov/pubmed/29602869

Consejo Nacional de Población [CONAPO]. (2016). Situación de la Salud Sexual y Reproductiva.

Recuperado de http://www.gob.mx/conapo

CONAPO, Secretaría de Gobernación, \& INMUJERES. (2016). Cartilla de derechos sexuales de $\begin{array}{lllll}\text { adolescentes } & \text { y } & \text { jóvenes } & \text { Recuperado }\end{array}$ https://www.cndh.org.mx/sites/all/doc/Programas/VIH/Divulgacion/cartillas/CartillaDerechos-Sexuales-Adolescentes-Jovenes.pdf 
Czuchry, M., Timpson, S., Williams, M. L., Bowen, A. M. \& Ratliff, E. A. (2009). Improving Condom Self-Efficacy and Use among Individuals Living with HIV: The Positive Choices Mapping Intervention. Journal of substance use, 14(3-4), 230-239. DOI: $10.1080 / 14659890902874212$

de Jesús-Reyes, D. \& Menkes-Bancet, C. (2014). Prácticas y significados del uso del condón en varones adolescentes de dos contextos de México. Papeles de Población, 20(79), 73-97.

de Jesús Reyes, D. \& Menkes-Bancet, C. (2017). Educación sexual y conductas sexuales en adolescentes escolarizados de Nuevo León (1a ed.). México: Miguel Ángel Porrúa.

Espada, J. P., Escribano, S., Morales, A. \& Orgilés, M. (2017). Two-year follow-up of a sexual health promotion program for Spanish adolescents. Evaluation and the Health Professions, 40(4), 483-504.

Fondo de Población de las Naciones Unidas México. (2018). Guía para la implementación de la estrategia nacional para la prevención del embarazo en adolescentes en las entidades federativas. UNFPA / UNICEF México. Recuperado de https://www.unicef.org/mexico/media/1816/file/Guia GEPEA.pdf

Gallegos, E. C., Villarruel, A. M., Loveland-Cherry, C., Ronis, D. L. \& Zhou, Y. (2008). Intervención para reducir riesgo en conductas sexuales de adolescentes: un ensayo aleatorizado y controlado. Salud Pública de México, 50, 59-66.

Gobierno de la República. (2016). Estrategia Nacional para la Prevención del Embarazo en Adolescentes. Ciudad de México.

Gómez-Inclán, S. \& Durán-Arenas, L. (2017). El acceso a métodos anticonceptivos en adolescentes de la Ciudad de México. Salud Pública de México, 59(3), 236.

Gutiérrez, J. P., Rivera-Dommarco, J., Shamah-Levy, T., Villalpando-Hernández, S., Franco, A., Cuevas-Nasu, L., Romero-Martínez, M \& Hernández-Ávila, M. (2012). Encuesta Nacional de Salud y Nutrición 2012. Resultados Nacionales. Recuperado de https://ensanut.insp.mx/informes/ENSANUT2012ResultadosNacionales.pdf

INEGI. (2015a). México en Cifras. Puebla. Recuperado de https://www.inegi.org.mx/app/areasgeograficas/?ag=21

INEGI. (2015b). México en cifras. Puebla indicadores. Recuperado de https://www.inegi.org.mx/app/areasgeograficas/?ag=21\#tabMCcollapse-Indicadores

INEGI. (2015c). Natalidad y fecundidad. Recuperado de 
Adaptación, aceptabilidad y pilotaje de "Usando Condón". Una intervención para incrementar la autoeficacia percibida del uso del condón masculino en adolescentes mexicanos

https://www.inegi.org.mx/app/tabulados/default.html?nc=mdemo29

INEGI. (2017). Población por edad y sexo. En Censos y conteos. Población y Vivienda. Recuperado de https://www.inegi.org.mx/temas/estructura/

INEGI. (2018a). Encuesta Nacional de la Dinámica Demográfica ENADID 2018. Recuperado de https://www.inegi.org.mx/contenidos/programas/enadid/2018/doc/resultados_enadid18.pdf

INEGI. (2018b). Natalidad y fecundidad. Recuperado de https://www.inegi.org.mx/temas/natalidad/

Kalamar, A. M., Bayer, A. M. \& Hindin, M. J. (2016). Interventions to Prevent Sexually Transmitted Infections, Including HIV, Among Young People in Low- and Middle-Income Countries: A Systematic Review of the Published and Gray Literature. Journal of Adolescent Health, 59(3), S22-S31.

Kelly, J. A., Heckman, T. G., Stevenson, L. Y., Williams, P. N., Ertl, T., Hays, R. B., Leonard, N. R., Terry, M. A., Sogolow, E. E. \& Neumann, M. S. (2000). Transfer of research-based HIV prevention interventions to community service providers: fidelity and adaptation. AIDS education and prevention: official publication of the International Society for AIDS Education, 12(5 Suppl), 87-98.

Lopez, L. M., Grey, T. W., Chen, M., Tolley, E. E. \& Stockton, L. L. (2016). Theory-based interventions for contraception. Cochrane Database of Systematic Reviews, 11(CD007249).

McKleroy, V. S., Galbraith, J. S., Cummings, B., Jones, P., Harshbarger, C., Collins, C., Gelaude, D., W. Carey, J. \& ADAPT Team. (2006). Adapting Evidence-Based Behavioral Interventions for New Settings and Target Populations. AIDS Education and Prevention, 18(supp.), 59-73. DOI: 10.1521/aeap.2006.18.supp.59

Morales, A., García-Montaño, E., Barrios-Ortega, C., Niebles-Charris, J., García-Roncallo, P., Abello-Luque, D., Gómez Lugo, M., Saavedra, D. A., Vallejo-Medina, P., Espada, J. P., Lightfoot, M. \& Martínez, O. (2019). Adaptation of an effective school-based sexual health promotion program for youth in Colombia. Social Science \& Medicine, 222, 207-215. DOI: https://doi.org/10.1016/j.socscimed.2019.01.011

OECD. (2019). Fertility rates (indicator). doi.org/10.1787/8272fb01-en

Protogerou, C., Hagger, M. S. \& Johnson, B. T. (2017). An integrated theory of condom use for young people in Sub-Saharan Africa: A meta-analysis. MetaArXiv.

Rogers, E. M. (2003). Diffusion of innovations (5 ed). Free Press. 
Sawyer, S. M., Azzopardi, P. S., Wickremarathne, D. \& Patton, G. C. (2018). The age of adolescence. The Lancet Child and Adolescent Health, 2(3), 223-228. DOI: 10.1016/S2352$\underline{4642(18) 30022-1}$

Secretaría de Salud. (2011). Métodos anticonceptivos temporales: DIU y condón. Consideraciones $\begin{array}{lllll}\text { de } & \text { uso } & \text { aplicación. } & \text { Recuperado }\end{array}$ http://www.cenetec.salud.gob.mx/descargas/gpc/CatalogoMaestro/505_GPC_DIUycondxn/ SS-505-11-EVR-DIU_CONDON.pdf

Straus, S. E., Tetroe, J. \& Graham, I. (2009). Defining knowledge translation. Canadian Medical Association Journal, 181(3-4), 165-168.

United Nations. (2016). The global strategy for women's, children's, and adolescents' health (2016-2030). Recuperado de https://www.who.int/life-course/partners/globalstrategy/globalstrategyreport2016-2030-lowres.pdf

Wang, T., Lurie, M., Govindasamy, D. \& Mathews, C. (2018). The effects of school-based condom availability programs (CAPs) on condom acquisition, use and sexual behavior: A systematic review. AIDS and Behavior, 22(1), 308-320. DOI: 10.1007/s10461-017-1787-5

WHO. (2016). Global health sector strategy on sexually transmitted infections 2016-2021. Towards ending. Recuperado de https://apps.who.int/iris/bitstream/handle/10665/246296/WHO-RHR-16.09eng.pdf;jsessionid=E9A67C7D523C49491189E94A281FA819?sequence $=1$

WHO. (2019). Sexually transmitted infections (STIs). Recuperado de https://www.who.int/en/news-room/fact-sheets/detail/sexually-transmitted-infections-(stis)

Widman, L., Noar, S. M., Choukas-Bradley, S. \& Francis, D. B. (2014). Adolescent sexual health communication and condom use: a meta-analysis. Health psychology: official journal of the Division of Health Psychology, American Psychological Association, 33(10), 1113-1124. DOI: $\underline{10.1037 / \text { hea0000112 }}$

World Health Organization [WHO]. (2017). Adolescent development. Recuperado de https://www.who.int/maternal_child_adolescent/topics/adolescence/development/en/ 